See discussions, stats, and author profiles for this publication at: https://www.researchgate.net/publication/333926647

\title{
Hydrogen Chemisorption on Doubly Vanadium Doped Aluminum Clusters
}

Article in Zeitschrift für Physikalische Chemie · June 2019

DOI: 10.1515/zpch-2019-1395

CITATIONS

0

9 authors, including:

Jan Vanbuel

KU Leuven

11 PUBLICATIONS 41 CITATIONS

SEE PROFILE

(2)

Mei-Ye Jia

Chinese Academy of Sciences

26 PUBLICATIONS 276 CITATIONS

SEE PROFILE

Some of the authors of this publication are also working on these related projects:

CITATIONS View project

Project PhD project View project
READS

186

Eva María Fernández

National Distance Education University

50 PUBLICATIONS 1,417 CITATIONS

SEE PROFILE

Piero Ferrari

KU Leuven

37 PUBlicATIONS 182 CITATIONS

SEE PROFILE 


\section{Jan Vanbuel, Eva M. Fernández, Mei-ye Jia, Piero Ferrari, Wieland Schöllkopf, Luis C. Balbás, Minh Tho Nguyen, André Fielicke and Ewald Janssens* Hydrogen Chemisorption on Doubly
Vanadium Doped Aluminum Clusters}

https://doi.org/10.1515/zpch-2019-1395

Received February 11, 2019; accepted March 27, 2019

Abstract: The interaction of hydrogen with doubly vanadium doped aluminum clusters, $\mathrm{Al}_{n} \mathrm{~V}_{2}{ }^{+}(n=1-12)$, is studied experimentally by time-of-flight mass spectrometry and infrared multiple photon dissociation spectroscopy. The hydrogen binding geometry is inferred from comparison with infrared spectra predicted by density functional theory and shows that for the more reactive clusters the hydrogen adsorbs dissociatively. Three sizes, $n=4,5$ and 7, are remarkably unreactive compared to the other clusters. For larger sizes the reactivity decreases, a behavior that is similar to that of singly vanadium doped aluminum clusters, and that might be attributed to geometric and/or electronic shielding of the dopants. By examining the electronic structure of $\mathrm{Al}_{6} \mathrm{~V}_{2}{ }^{+}$and $\mathrm{Al}_{7} \mathrm{~V}_{2}{ }^{+}$, interactions between the frontier orbitals of the clusters and those of $\mathrm{H}_{2}$ that explain the size-dependent reactivity are identified.

Keywords: density functional theory calculations; hydrogen storage; ionmolecule reactions; IR spectroscopy; mass spectrometry; metal clusters.

*Corresponding author: Ewald Janssens, Laboratory of Solid-State Physics and Magnetism, KU Leuven, 3001 Leuven, Belgium, e-mail: ewald.janssens@kuleuven.be

Jan Vanbuel, Mei-ye Jia and Piero Ferrari: Laboratory of Solid-State Physics and Magnetism, KU Leuven, 3001 Leuven, Belgium

Eva M. Fernández: Departamento de Física Fundamental, Universidad Nacional de Educación a Distancia, 28040 Madrid, Spain

Wieland Schöllkopf and André Fielicke: Fritz-Haber-Institut der Max-Planck-Gesellschaft, 14195 Berlin, Germany

Luis C. Balbás: Departamento de Física Teórica, Universidad de Valladolid, 47011 Valladolid, Spain

Minh Tho Nguyen: Department of Chemistry, KU Leuven, 3001 Leuven, Belgium 


\section{Introduction}

Hydrogen can be used to buffer the intermittent nature of renewable energy sources (RES) such as solar and wind power. When electricity production from RES exceeds demand, this excess can be used to electrolyze water into hydrogen and oxygen; the hydrogen is then stored and can later, when needed, be re-electrified in a fuel cell [1]. To increase the role of hydrogen in the energy transition, technological advances in production, storage and re-electrification of hydrogen are needed. Solid state storage, i.e. the storage of hydrogen in chemical compounds, is a safe, energy-efficient and compact alternative to the liquid and high-pressure storage methods that are currently in use. Promising storage media are hydrides of light and abundant metals and complex metal hydrides such as alane, $\mathrm{AlH}_{3}$, and sodium alanate, $\mathrm{NaAlH}_{4}$, which have hydrogen weight percentages of $10 \%$ and $7 \%$, respectively [2]. Unfortunately, these materials are difficult to form due to high activation barriers, a property which has been observed for bulk as well as nanosized aluminum. Bogdanović and Schwickardi discovered that titanium doping activates aluminum for reversible hydrogen adsorption [3]. Moreover, not only Ti, but also other light transition metals such as Sc and V have been shown to catalyze the hydrogenation of sodium alanate [4].

Although hydrogen storage materials are mainly studied as bulk solids, their chemistry occurs at the molecular scale. Small clusters, particles consisting of a few to tens of atoms, are therefore useful model systems, allowing for the identification and elucidation of possible reaction pathways and catalytically active sites. Gas-phase experiments enable the simultaneous study of a wide size range of clusters under well-defined experimental conditions. Moreover, by studying these clusters in the gas phase instead of deposited on a surface, one can separate the intrinsic cluster properties from support-induced properties, which significantly reduces the complexity of the computational modelling that is required for a detailed understanding of their chemistry $[5,6]$.

Recent combined experimental and computational studies of singly vanadium and singly and doubly rhodium doped aluminum clusters [7-9] showed that hydrogen can chemisorb dissociatively, as well as molecularly via the Kubas interaction [10]. Such molecular side-on bound $\mathrm{H}_{2}$-complexes on transition metal atoms have been first described by Kubas and termed 'non-classical' in contrast to the 'classical' complexes containing atomic H-ligands. The binding model parallels the Dewar-Chatt-Duncanson model for $\pi$-complexes but explains the 3 center - 2 electron $\sigma$-bonding between $\mathrm{H}_{2}$ and transition metal atoms [4, 5]: the interaction is governed by donation of electron density from the bonding $\sigma$ orbital of $\mathrm{H}_{2}$ (HOMO) into an empty d-orbital of the metal atom, and back-donation from 
an occupied d-orbital into the empty $\sigma^{*}$ orbital (LUMO) of $\mathrm{H}_{2}$. This model not only explains the stability of the non-classical $\mathrm{H}_{2}$-complexes, but also describes a mechanism for the homolytic splitting of the $\mathrm{H}-\mathrm{H}$ bond.

For the singly vanadium doped aluminum clusters, $\mathrm{Al}_{n} \mathrm{~V}^{+}$, only a limited range of sizes, i.e. $n=2$ and $n \geq 10$, were reactive towards hydrogen. Previous work on rhodium doped clusters showed that the doubly doped species are more reactive than the singly doped ones $[8,9]$. Also for Ti-doped sodium alanate, computational studies suggest that the active site consists of at least two neighboring Ti atoms [11].

In this work, we report on the interaction of hydrogen with doubly vanadium doped aluminum clusters. Structural information about the hydrogen binding geometry is obtained via infrared action spectroscopy experiments in combination with density functional theory (DFT) calculations. Analysis of the density of states and the molecular orbitals of the cluster- $\mathrm{H}_{2}$ complex gives insight into the chemical bonding of the complex and the existence or absence of activation barriers along the kinetic reaction pathway.

\section{Materials and methods}

\subsection{Experimental}

$\mathrm{Al}_{n} \mathrm{~V}_{2}{ }^{+}(n=1-12)$ clusters are produced in a dual laser (Nd-YAG, Continuum Minilite, $10 \mathrm{~Hz}, \lambda=532 \mathrm{~nm}, t_{\text {pulse }} \approx 6 \mathrm{~ns}$ ) ablation source, which is described in more detail in ref. [12]. After production in the source, the molecular beam is collimated by a skimmer before entering the extraction zone, after which the clusters are detected in an orthogonal time-of-flight mass spectrometer. The hydrogen gas is injected through a separate valve into an extension channel of the cluster source, before expansion of the molecular beam into the vacuum. To infer the hydrogen binding geometry, infrared multiple photon dissociation (IRMPD) action spectroscopy is performed at the free electron laser (FEL) facility of the Fritz-Haber-Institut [13] in the $800-2100 \mathrm{~cm}^{-1}$ wavenumber range with an average pulse energy of approximately $50 \mathrm{~mJ}$. The FEL beam is focused through a $1 \mathrm{~mm}$ aperture a few $\mathrm{cm}$ upstream of the extraction region of the mass spectrometer, defining the spatial overlap between cluster and infrared (IR) laser beams. When the FEL frequency is in resonance with an IR active vibrational mode of a cluster complex, it heats up via internal vibrational redistribution [14]. If the internal energy of a complex gets sufficiently high to lead to dissociation, the abundance of this complex in the mass spectrum depletes. For all complexes that 
depleted upon FEL irradiation, the observed decay channel was the loss of $\mathrm{H}_{2}$ molecules. Parts of mass spectra measured with and without FEL irradiation can be found in the supporting information (SI).

\subsection{Computational}

DFT calculations are performed by employing the Gaussian 09 software package [15] with the generalized gradient approximated (GGA) exchange correlation functional as devised by Perdew, Burke, and Ernzerhof (PBE) [16]. This functional was previously adopted in our work for $\mathrm{H}_{2}$ adsorption on singly vanadium doped and singly and doubly rhodium doped aluminum clusters [7-9]. Global optimizations were carried out for $\mathrm{Al}_{n} \mathrm{~V}_{2}{ }^{+}(n=1-12)$ clusters and their hydrogenated counterparts $\mathrm{Al}_{n} \mathrm{~V}_{2} \mathrm{H}_{2}{ }^{+}$by the CALYPSO [17] methodology. The low-lying isomers were collected and optimized for a range of possible spin multiplicities with the Def2TZVP [18] basis set. In addition to searching by CALYPSO, we manually built various initial structures for $\mathrm{Al}_{n} \mathrm{~V}_{2}{ }^{+}$clusters based on pure vanadium and aluminum counterparts. For $\mathrm{Al}_{n} \mathrm{~V}_{2} \mathrm{H}_{2}{ }^{+}$, a large number of structures were constructed and tested by adding two $\mathrm{H}$ atoms or one $\mathrm{H}_{2}$ molecule to the low-energy isomers of $\mathrm{Al}_{n} \mathrm{~V}_{2}{ }^{+}$. Various orientations have been considered for the bonded $\mathrm{H}$ atoms (terminal, bridging, and facet sites) and $\mathrm{H}_{2}$ molecule (end-on and side-on). At the PBE/Def2TZVP level, harmonic and anharmonic vibrational frequency calculations were performed to simulate the IR spectra and to obtain the zero-point energy (ZPE) corrections for the electronic energies. The vibrational frequencies are unscaled. To calculate $\mathrm{H}_{2}$ adsorption energies at the PBE/ Def2TZVP level, we employed the D3 version of Grimme's dispersion corrections with Becke-Johnson damping (GD3BJ) [19].

\section{Results and discussion}

Reactions of vanadium doped aluminum clusters with $\mathrm{H}_{2}$ are studied by adding hydrogen gas under multiple collision conditions to the thermalized clusters within the reaction channel of the cluster source. Figure 1a shows a representative mass spectrum of the vanadium doped aluminum clusters with (in red) and without (in black) $\mathrm{H}_{2}$ gas injected into the source. The inset contains a close-up of a part of the mass spectrum, showing some of the hydrogenated complexes. Complexes of aluminum clusters containing one to three vanadium atoms can be identified. The hydrogenated clusters containing a single $\mathrm{V}$ atom have been discussed in ref. [7]. Here we will discuss only the doubly doped clusters and the effects emerging from the presence of the two, as will be shown below, directly 


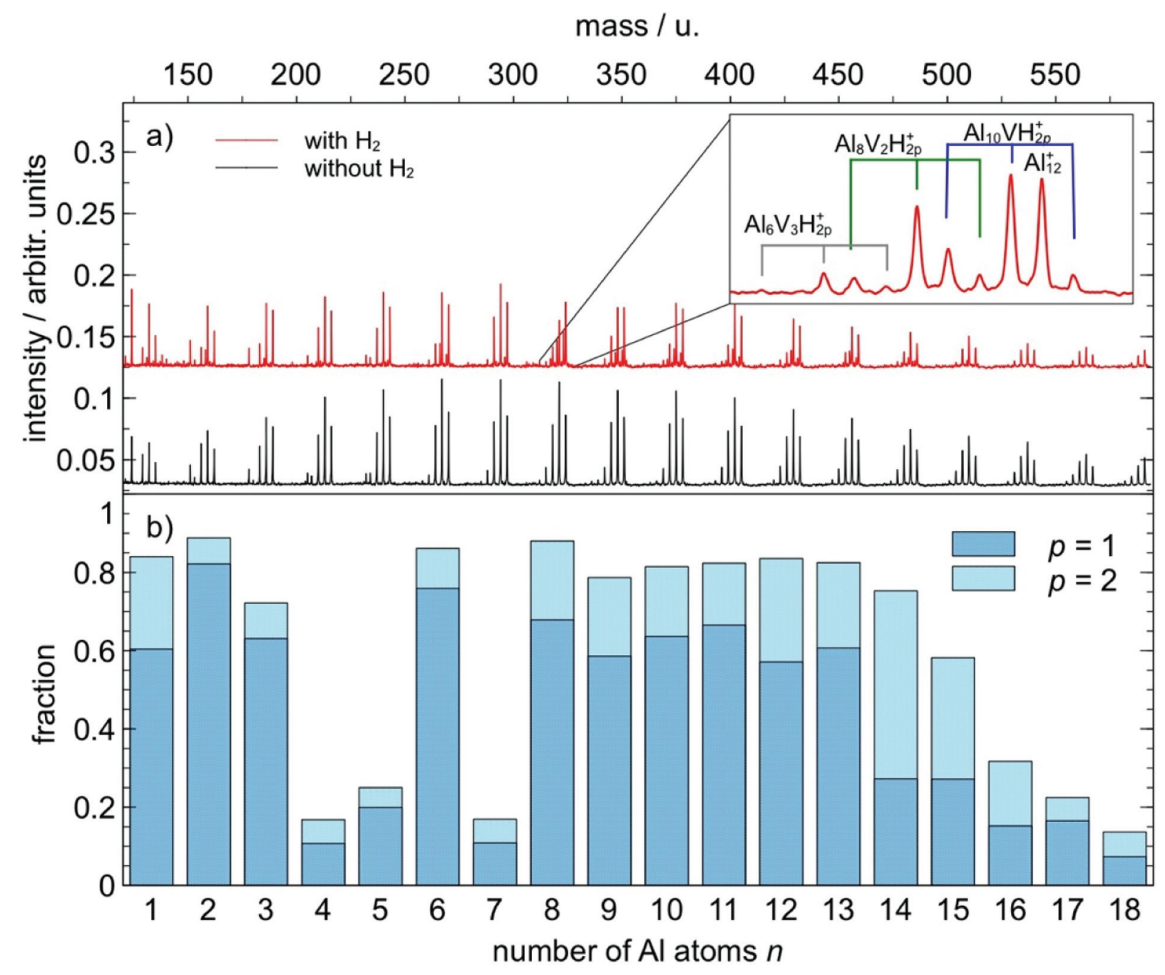

Fig. 1: (a) Mass spectrum of $\mathrm{Al}_{n} \mathrm{~V}_{m}+(n=1-18, m=0-3)$ clusters, with and without hydrogen, together with an inset showing part of the abundances of the individual hydrogenated clusters, and (b) fractional distribution of $\mathrm{Al}_{n} \mathrm{~V}_{2} \mathrm{H}_{2 p}+(n=1-18, p=1-2)$ clusters.

neighbored $\mathrm{V}$ atoms. For these clusters, $\mathrm{Al}_{n} \mathrm{~V}_{2}{ }^{+}(n=1-18)$, the fractional distribution of hydrogenated complexes is plotted in Figure $1 \mathrm{~b}$, which shows the fraction $F\left(\mathrm{H}_{2 p}\right)$ of $\mathrm{Al}_{n} \mathrm{~V}_{2}{ }^{+}$that adsorb $p$ (with $p=1$ or 2 ) hydrogen molecules, and which is defined as

$$
F\left(\mathrm{H}_{2 p}\right)=\frac{I\left(\mathrm{Al}_{n} \mathrm{~V}_{2} \mathrm{H}_{2 p}^{+}\right)}{\sum_{i=0-2} I\left(\mathrm{Al}_{n} \mathrm{~V}_{2} \mathrm{H}_{2 i}^{+}\right)} .
$$

Most $\mathrm{Al}_{n} \mathrm{~V}_{2}{ }^{+}$clusters adsorb a single hydrogen molecule. Only for $n=14$ and 15 , clusters with $p=2$ are more abundant than the singly hydrogenated species. Three sizes, $n=4,5$ and 7, are remarkably unreactive compared to their neighboring sizes. For larger sizes, the reactivity decreases. This decrease in reactivity for larger sizes has also been observed for singly vanadium doped clusters and 
has been attributed to cage formation [9, 20, 21]. For the singly doped clusters, the onset of cage formation, i.e. the size at which the vanadium dopant occupies an endohedral instead of exohedral position in the cluster, occurs at $n=16$. Here, however, the decrease is seen after $n=15$. A possible explanation could be that at this size, one of the vanadium atoms occupies an endohedral position, and the other is partially shielded, either geometrically [21] or electronically [22].

Understanding the size-dependence of the reactivity of transition metal (doped) clusters in the gas phase is generally not an easy task due to the possibility of low-lying geometric or electronic states (possibly even of a different multiplicity), whose potential energy surfaces (PES) during the reaction with hydrogen might cross that of the ground state. Nevertheless, complementing the experimental data with ab initio calculations of the thermodynamics of the reaction often goes a long way in explaining the observations $[8,9]$. Furthermore, one can identify features in the electronic structure, which reveal why some clusters react strongly with a given reagent, and why others do not [7].

For the present discussion we focus on two local minima that are typically present on the PES of the cluster-hydrogen interaction. The first is a complex with molecularly adsorbed hydrogen. Our previous results have shown that the molecular adsorption of hydrogen on transition metal doped aluminum clusters occurs without barrier and the molecular complex can be bound quite strongly via the Kubas interaction or more weakly via van-der-Waals interactions $[8,9]$. Another possibility is that the hydrogen dissociates into atomically bound hydrogen, a configuration which is possibly separated from the molecular local minimum by an activation barrier [7]. In order to detect the reaction products via mass spectrometry, it is necessary for these products to be stable on the timescale of the experiment, or more precisely, during the time the clusters spend between the source and the extraction zone of the time-of-flight mass spectrometer, which is on the order of $100 \mu \mathrm{s}$. For reactions at room temperature, the possibility of detecting physisorbed complexes is excluded due to their low binding energy. Assuming a cluster temperature close to $300 \mathrm{~K}$, the temperature of the source, physisorbed complexes are estimated by statistical models to decay on much faster timescales, i.e. a few $\mu \mathrm{s}$ at most. The hydrogenated clusters that are detected in our experiment are therefore more likely to be Kubas-complexes or clusters with dissociatively bound hydrogen, in line with our earlier findings for singly vanadium doped and singly and doubly rhodium doped aluminum clusters [7-9].

Shown in Figure 2 are the IRMPD spectra for the hydrogenated $\mathrm{Al}_{n} \mathrm{~V}_{2}{ }^{+}$clusters $(n=2,3,6,8-12)$, together with the calculated infrared spectra of the lowestenergy isomer found via the global PES search. For $n=4,5$ and 7, the unreactive sizes, the signal-to-noise ratio of the IRMPD spectra was not high enough to clearly discern absorption bands. The experimental IRMPD spectrum $Y(v)$ is defined as 


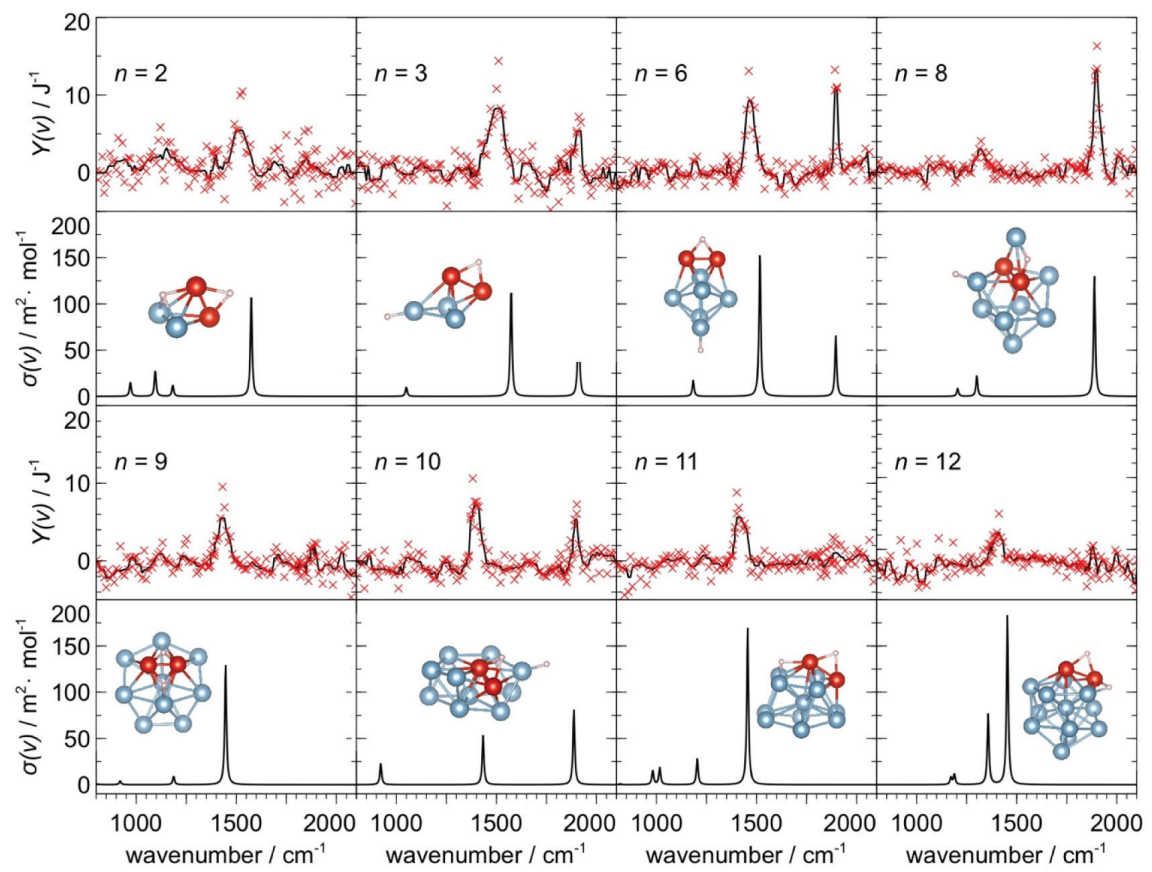

Fig. 2: Infrared spectra of $\mathrm{Al}_{n} \mathrm{~V}_{2} \mathrm{H}_{2}+(n=2,3,6,8-12)$. First and third row: experimental IRMPD spectra. Second and fourth row: DFT predicted IR spectra of the obtained lowest energy isomers. The calculated IR stick spectra are artificially broadened by $\Gamma=10 \mathrm{~cm}^{-1}$ to facilitate comparison with the experimental spectra and therefore have the unit of a cross section. The spectra are then, scaled by a factor of $10 \sqrt{2 \pi}$ for the maxima to be comparable to literature values in $\mathrm{km} / \mathrm{mol}$.

$$
Y(v)=-\frac{\log \left(\frac{I_{\mathrm{FEL}}}{I_{0}}\right)}{E(v)}
$$

with $I_{\mathrm{FEL}} / I_{0}$ the abundance of the cluster with/without the FEL crossing the molecular beam and $E(v)$ the energy per pulse of the FEL. Although the quantity $Y(v)$ often qualitatively agrees with calculated single photon absorption cross sections [23, 24], significant differences can arise due to multiple photon nature, the role of anharmonic coupling, and the statistical dissociation involved in the IRMPD process [25-27]. For most clusters, the agreement with the IR spectra of the putative lowest energy structure is quite good. The main features in the experimental spectra are bands around $1500 \mathrm{~cm}^{-1}$ and at the high end (at $\left.1900 \mathrm{~cm}^{-1}\right)$. The first band corresponds to both the symmetric and asymmetric stretching mode of a hydrogen atom bridging two vanadium atoms or bridging an $\mathrm{Al}-\mathrm{V}$ bond. The band at $1900 \mathrm{~cm}^{-1}$ has also been observed for the other transition metal 
doped aluminum clusters and corresponds to the stretching mode of a hydrogen atom bound on-top of a single aluminum atom [7, 8]. Thus, for all reactive clusters, the IRMPD spectra strongly suggest the presence of atomic hydrogen, or in other words that the hydrogen adsorbs dissociatively. Also, for all lowest energy isomers, at least one of the hydrogen atoms is bound to one or two vanadium atoms. As the d-orbitals of the vanadium are mainly responsible for activating the $\mathrm{H}-\mathrm{H}$ bond, the atomic hydrogen bound to one or two of the vanadium dopants could be seen as 'poisoning' the dopants and this explains why most clusters have only a single hydrogen molecule adsorbed. Moreover, the IRMPD spectra of $\mathrm{Al}_{14} \mathrm{~V}_{2} \mathrm{H}_{2}{ }^{+}$and $\mathrm{Al}_{15} \mathrm{~V}_{2} \mathrm{H}_{2}{ }^{+}$(SI) do not have the bands that are characteristic for the (a)symmetric stretch of $\mathrm{Al}-\mathrm{H}-\mathrm{V} / \mathrm{V}-\mathrm{H}-\mathrm{V}$ bonds, suggesting that for these sizes the hydrogen does not remain bound to the vanadium but spills over to the aluminum. Although the interpretation of these infrared spectra is more difficult because of intensity ingrowth coming from depletion of the clusters with two $\mathrm{H}_{2}$ molecules, this observation is in line with the poisoning hypothesis. It could explain why a large fraction of the $n=14,15$ clusters have one more hydrogen molecule adsorbed than the other clusters (Figure 1b). For an overview of the lowest energy structures for all clusters, also those that are unreactive, we refer to Figure S3 in the SI.

To gain a better understanding of the size-dependent reactivity, the thermodynamics of the reaction was calculated with DFT (for an overview of calculated properties, see Table S1 in the SI). Plotted in Figure 3 are (a) the experimentally observed total fraction of hydrogenated clusters $\Sigma F\left(\mathrm{H}_{2 p}\right)$, (b) the molecular hydrogen binding energy $\mathrm{BE}\left(\mathrm{H}_{2}\right)$, (c) the dissociative binding energy $\mathrm{BE}(2 \mathrm{H})$ and (d) the $\mathrm{H}-\mathrm{H}$ bond distance in the respective putative most stable isomers of the $\mathrm{Al}_{n} \mathrm{~V}_{2}{ }^{+} \cdot\left(\mathrm{H}_{2}\right)$ clusters, i.e. with the hydrogen molecularly adsorbed. An overview of the structures of the bare $\mathrm{Al}_{n} \mathrm{~V}_{2}{ }^{+}$clusters, as well as the $\mathrm{Al}_{n} \mathrm{~V}_{2}{ }^{+}$clusters with molecularly and dissociatively adsorbed hydrogen can be found in the SI (Figure S3). Note that for some sizes, e.g. $n=4$ and 8 , there is a considerable change in geometry going from the molecularly adsorbed lowest-energy isomer to the dissociatively adsorbed structure. Although such structural changes are often accompanied by an energy barrier [28], theoretical calculations indicate that for aluminum clusters these barriers can be offset by the adsorption energy of dissociatively adsorbed hydrogen [29].

From Figure 3 it can be seen that there is some correlation between the molecular binding energy, the $\mathrm{H}-\mathrm{H}$ bond distance and the experimental abundances. The imperfect correlation could be due to an incorrect assignment of the ground state predicted by the DFT calculations. For the smaller sizes, $n=1-5$, we found slightly different lowest energy structures at the same level of theory but calculated with the SIESTA [30] code, which uses a numerical pseudo-atomic basis set 


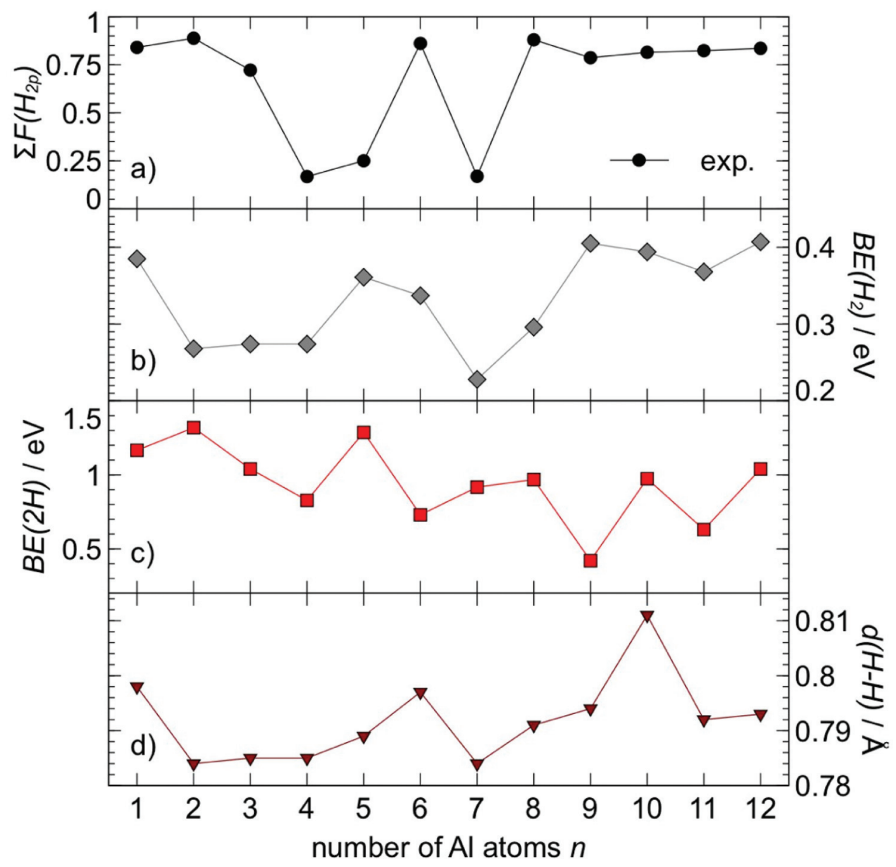

Fig. 3: (a) Experimental total fraction of hydrogenated $\mathrm{Al}_{n} \mathrm{~V}_{2}+(n=1-12)$ clusters, (b) molecular $\mathrm{H}_{2}$ binding energy, (c) dissociative $2 \mathrm{H}$ binding energy, (d) $\mathrm{H}-\mathrm{H}$ bond length of the molecularly adsorbed hydrogen.

[31] instead of a Gaussian basis set. For $n=2$, for example, the lowest energy isomer predicted by SIESTA has a higher molecular $\mathrm{H}_{2}$ binding energy, as well as more activated $\mathrm{H}_{2}$ bond. The structures and binding energies of these isomers can be found in Figure S4 and Table 2 of the SI. A similar correlation between the molecular binding energy, the $\mathrm{H}-\mathrm{H}$ distance and the experimental abundances was observed for the rhodium doped aluminum clusters [9], for which it was argued that the rate-limiting step of the reaction was the formation of the $\mathrm{H}_{2}$-cluster complex, although the final state as evidenced by infrared spectroscopy was the thermodynamically most favorable configuration $[8,9]$. Transition metal clusters have also been shown to chemisorb hydrogen dissociatively via a precursor-mediated reaction pathway [32]. Conceicao et al. [33] also found an inverse correlation between the reactivity of transition metal clusters and the energy needed for promoting an electron to a higher lying energy level which does not have the same symmetry as the $\sigma$ orbital as hydrogen, thereby reducing the Pauli repulsion energy. Heuristically, the link between the precursor and promotion energy model is that clusters with a higher density of states at the Fermi level 
have both a low promotion energy cost as well as higher polarizability, explaining both the decreased activation barrier and the increased stability of the precursor complex [32,33]. Although these models are convincing in their generality and applicability to a broad range of transition metal clusters, a detailed analysis of the electronic structure should be used to verify their predictions.

For $n=7$, the binding energy of the molecularly adsorbed $\mathrm{H}_{2}$ is very low, only $0.23 \mathrm{eV}$, indicating that the interaction is rather physical (ion-induced dipole) than chemical. This can also be seen in the $\mathrm{H}-\mathrm{H}$ distance, which shows little sign of activation compared to the calculated bond length of free $\mathrm{H}_{2} d(\mathrm{H}-\mathrm{H})=0.75 \AA$. Looking at the electronic density of states (DOS) of $\mathrm{Al}_{7} \mathrm{~V}_{2}{ }^{+}$and $\mathrm{Al}_{7} \mathrm{~V}_{2}{ }^{+} \cdot\left(\mathrm{H}_{2}\right)$ in Figure $4 \mathrm{a}$ and $\mathrm{b}$, respectively, there are only three energy levels of the bare cluster which after the $\mathrm{H}_{2}$ complex formation have visible $\mathrm{H}(\mathrm{s})$-character, one at low energy $(-13.3 \mathrm{eV})$, and two above the HOMO level and therefore unoccupied (around $-2 \mathrm{eV}$ ). The orbital at $-13.3 \mathrm{eV}$ corresponds to the bonding $\sigma$ orbital of
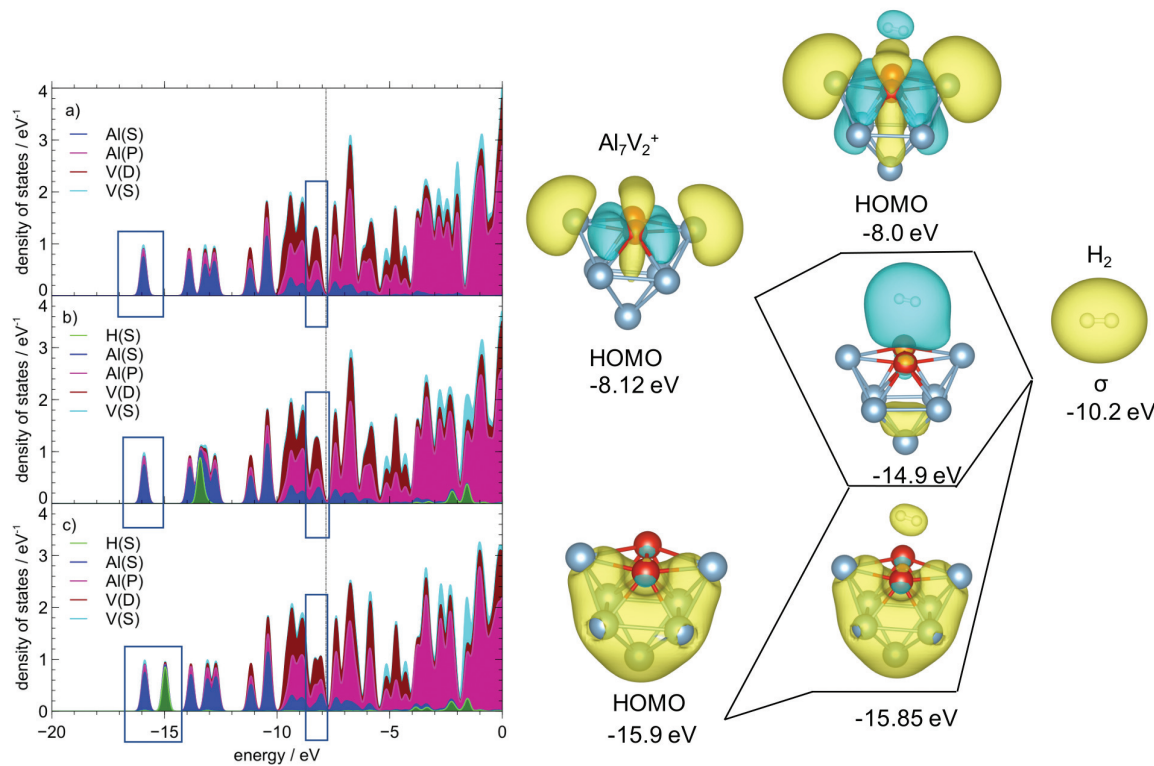

Fig. 4: Left: density of states of (a) $\mathrm{Al}_{7} \mathrm{~V}_{2}+$ and (b) $\mathrm{Al}_{7} \mathrm{~V}_{2}+\cdot\left(\mathrm{H}_{2}\right)$. The levels left of the dotted line are occupied, the ones to the right are unoccupied. The occupied energy level in (b) at $-13.3 \mathrm{eV}$ consists mainly of the $\sigma\left(\mathrm{H}_{2}\right)$ orbital, whereas the two unoccupied states of $\mathrm{H}(\mathrm{S})$ character around $-2 \mathrm{eV}$ correspond to the anti-bonding $\sigma^{*}\left(\mathrm{H}_{2}\right)$. (c) Density of states with $\mathrm{H}_{2}$ placed $0.9 \AA$ A closer to the cluster. The cluster levels that shift around the Fermi level are being repelled by the $\sigma$ orbital of $\mathrm{H}_{2}$. Right: schematic representation of the relevant orbitals participating in the reaction between $\mathrm{Al}_{7} \mathrm{~V}_{2}+$ and $\mathrm{H}_{2}$ when the hydrogen is placed $0.9 \AA$ closer to the cluster than in the equilibrium position. Those orbitals correspond to the energy levels framed by the black boxes in (a) and (c). 
$\mathrm{H}_{2}$, the other two are mainly of $\sigma^{*}$-character. For the levels that lie in between these extremes, especially the ones close to the HOMO level, the hydrogen orbitals only contribute a small percentage to the complex orbitals, but its these orbitals that matter. Because there is not much overlap in their respective orbitals, the interaction between $\mathrm{H}_{2}$ and $\mathrm{Al}_{7} \mathrm{~V}_{2}{ }^{+}$is very weak. To help understand the weak interaction between the two, Figure 4c illustrates the change in the DOS when the hydrogen molecule is pushed from its equilibrium position, about 3.5 Å above the $\mathrm{V}_{2}$ unit, 0.9 Å closer to the cluster. It can be seen that the HOMO level slightly shifts up in energy by $0.12 \mathrm{eV}$ due to Pauli repulsion as shown schematically on the right side of Figure 4. The corresponding energy levels in the DOS are highlighted by the black frames. Besides the HOMO orbital there are a few other orbitals that exhibit the same behavior, i.e. a small upwards shift due to Pauli repulsion. None of the occupied orbitals mix noticeably with the $\sigma^{*}$ orbital of $\mathrm{H}_{2}$, explaining the non-activated $\mathrm{H}-\mathrm{H}$ bond and the low binding energy which is mainly due to ion-induced electrostatic interaction and dispersion forces instead of chemical bonding.

For $\mathrm{Al}_{6} \mathrm{~V}_{2}{ }^{+}$, which binds $\mathrm{H}_{2}$ more strongly, the main interaction between the cluster and the hydrogen molecule is not via the $\sigma$ orbital of $\mathrm{H}_{2}$ (although these orbitals are also present below $-15 \mathrm{eV}$ in the DOS of Figure 5, having a high percentage of $\mathrm{H}(\mathrm{S})$-character), but between the $\sigma^{*}$ orbital of $\mathrm{H}_{2}$ and the occupied cluster orbitals near the Fermi level (the levels within the black frames). As shown
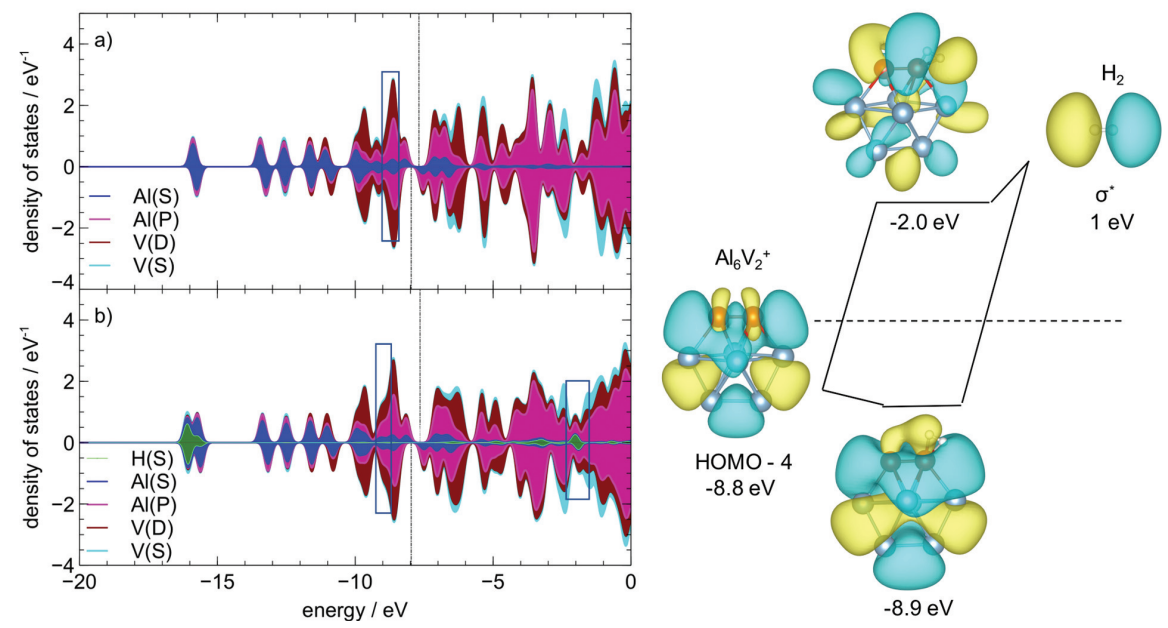

Fig. 5: Left: density of states of (a) $\mathrm{Al}_{6} \mathrm{~V}_{2}+$ and (b) $\mathrm{Al}_{6} \mathrm{~V}_{2}+\cdot\left(\mathrm{H}_{2}\right)$. The levels left of the dotted line are occupied, the ones to the right are unoccupied. The positive and negative y-axis corresponds to spin-up and spin-down electrons, respectively. Right: schematic representation of the relevant orbitals participating in the reaction between $\mathrm{Al}_{6} \mathrm{~V}_{2}+$ and $\mathrm{H}_{2}$. 
on the right side of Figure 5, one of the filled cluster orbitals donates electrons to the initially empty $\sigma^{*}$. This interaction weakens the $\mathrm{H}-\mathrm{H}$ bond, and thereby reduces the activation barrier for dissociative adsorption.

Compared to the singly vanadium doped clusters [7], the doubly doped clusters are much more reactive (based of the total fraction $\Sigma \mathrm{F}\left(\mathrm{H}_{2 p}\right)$ of hydrogenated clusters, see Figure S5 in the SI with both fractional distributions obtained from the same mass spectrum). Nevertheless, the $\mathrm{H}_{2}$ activation as measured by the $\mathrm{H}-\mathrm{H}$ distance in the physisorbed state is less than was the case for the singly doped clusters. The molecular binding energies, on the other hand, are slightly larger. A possible explanation for the weakened molecular activation can be found in the binding location of the $\mathrm{H}_{2}$ molecule: it exclusively binds on the periphery of the two vanadium atoms (Figure S3), as the bond between the two is a strong $\sigma$-bond, which is repulsive for the HOMO of hydrogen. However, assuming that a molecularly bound precursor state is rate-determining, a second vanadium dopant does increase the probability of a collision resulting in such a precursor state. This could also explain why for smallest sizes there is no correlation between calculated $\mathrm{d}(\mathrm{H}-\mathrm{H})$ and $\mathrm{BE}\left(\mathrm{H}_{2}\right)$ with the abundance of the hydrogenated clusters; for those sizes, the higher ratio of $\mathrm{V}$ to $\mathrm{Al}$ makes an effective collision resulting in a molecularly bound complex more likely.

\section{Conclusion}

In summary, the interaction of hydrogen with doubly vanadium doped aluminum clusters, $\mathrm{Al}_{n} \mathrm{~V}_{2}{ }^{+}(n=1-12)$, is studied experimentally by time-of-flight mass spectrometry and infrared multiple photon dissociation spectroscopy. The hydrogen binding geometry is inferred from comparison with infrared spectra predicted by density functional theory and shows that for all reactive clusters the hydrogen adsorbs dissociatively. All reactive clusters have a band around $1500 \mathrm{~cm}^{-1}$, corresponding to the vibration of a hydrogen atom bridging a $\mathrm{V}-\mathrm{V}$ and/or $\mathrm{V}-\mathrm{Al}$ bond. This suggests poisoning of the transition metal site, and could explain the limited number of hydrogen molecules adsorbed. There is a reasonable correlation between the molecular binding energy $\mathrm{BE}\left(\mathrm{H}_{2}\right)$, the distance between the two hydrogen atoms in the molecularly adsorbed geometry, $\mathrm{d}(\mathrm{H}-\mathrm{H})$, and the experimentally observed abundance. By examining the electronic structure of two representative clusters, i.e. the reactive $n=6$ and the unreactive $n=7$, the observed (un)reactivity can be explained by the presence (absence) of cluster orbitals that interact with the anti-bonding $\sigma^{*}$ orbital of hydrogen, which not only results in a higher molecular binding energy but also weakens the strong $\mathrm{H}-\mathrm{H}$ bond and facilitates dissociative adsorption. 
Acknowledgments: This work is supported by the KU Leuven Research Council (Funder Id: http://dx.doi.org/10.13039/501100004497, C1/18/073) and by the Research Foundation Flanders (FWO, Funder Id: http://dx.doi.org/10.13039/ 501100003130, Project G.0B41.15N). J.V. and P.F. thank the FWO for a PhD fellowship and a postdoctoral fellowship, respectively. E.M.F. thanks the RyC contract (ref. RYC-2014-15261) of the Spanish Minister of Economy, Industry and Competitiveness. A.F. thanks the Deutsche Forschungsgemeinschaft for a Heisenberg grant (Funder Id: http://dx.doi.org/10.13039/501100001659, FI893/5).

\section{References}

1. M. Ball, M. Weeda, Int. J. Hydrogen Energy 40 (2015) 7903.

2. F. Schüth, B. Bogdanović, M. Felderhoff, Chem. Commun. 0 (2004) 2249.

3. B. Bogdanović, M. Schwickardi, J. Alloys Compd. 253-254 (1997) 1.

4. Y. Liu, Z. Ren, X. Zhang, N. Jian, Y. Yang, M. Gao, H. Pan, Energy Technol. 6 (2018) 487.

5. H. Schwarz, Angew. Chem. Int. Ed. 54 (2015) 10090.

6. S. M. Lang, T. M. Bernhardt, Phys. Chem. Chem. Phys. 14 (2012) 9255.

7. J. Vanbuel, E. M. Fernandez, P. Ferrari, S. Gewinner, W. Schöllkopf, L. C. Balbas, A. Fielicke, E. Janssens, Chem. Eur. J. 23 (2017) 15638.

8. J. Vanbuel, M. Jia, P. Ferrari, S. Gewinner, W. Schöllkopf, M. T. Nguyen, A. Fielicke, E. Janssens, Top. Catal. 61 (2018) 62-70.

9. M. Jia, J. Vanbuel, P. Ferrari, E. M. Fernández, S. Gewinner, W. Schöllkopf, M. T. Nguyen, A. Fielicke, E. Janssens, J. Phys. Chem. C 122 (2018) 18247.

10. G. J. Kubas, Chem. Rev. 107 (2007) 4152.

11. S. Chaudhuri, J. Graetz, A. Ignatov, J. J. Reilly, J. T. Muckerman, J. Am. Chem. Soc. 128 (2006) 11404.

12. N. X. Truong, M. Haertelt, B. K. A. Jaeger, S. Gewinner, W. Schöllkopf, A. Fielicke, O. Dopfer, Int. J. Mass Spectrom. 395 (2016) 1-6.

13. W. Schöllkopf, S. Gewinner, H. Junkes, A. Paarmann, G. von Helden, H. Bluem, A. M. M. Todd, Proc. SPIE 9512 (2015) 95121L.

14. D. J. Nesbitt, R. W. Field, J. Phys. Chem. 100 (1996) 12735.

15. M. J. Frisch, G. W. Trucks, H. B. Schlegel, G. E. Scuseria, M. A. Robb, J. R. Cheeseman, G. Scalmani, V. Barone, G. A. Petersson, H. Nakatsuji, X. Li, M. Caricato, A. Marenich, J. Bloino, B. G. Janesko, R. Gomperts, B. Mennucci, H. P. Hratchian, J. V. Ortiz, A. F. Izmaylov, J. L. Sonnenberg, D. Williams-Young, F. Ding, F. Lipparini, F. Egidi, J. Goings, B. Peng, A. Petrone, T. Henderson, D. Ranasinghe, V. G. Zakrzewski, J. Gao, N. Rega, G. Zheng, W. Liang, M. Hada, M. Ehara, K. Toyota, R. Fukuda, J. Hasegawa, M. Ishida, T. Nakajima, Y. Honda, O. Kitao, H. Nakai, T. Vreven, K. Throssell, J. A. Montgomery, Jr., J. E. Peralta, F. Ogliaro, M. Bearpark, J. J. Heyd, E. Brothers, K. N. Kudin, V. N. Staroverov, T. Keith, R. Kobayashi, J. Normand, K. Raghavachari, A. Rendell, J. C. Burant, S. S. Iyengar, J. Tomasi, M. Cossi, J. M. Millam, M. Klene, C. Adamo, R. Cammi, J. W. Ochterski, R. L. Martin, K. Morokuma, O. Farkas, J. B. Foresman, D. J. Fox, Gaussian 09, Revision E.01, Gaussian, Inc., Wallingford CT (2016).

16. J. P. Perdew, K. Burke, M. Ernzerhof, Phys. Rev. Lett. 77 (1996) 3865. 
17. H. Wang, Y. Wang, J. Lv, Q. Li, L. Zhang, Y. Ma, Comput. Mater. Sci. 112 (2016) 406.

18. F. Weigend, R. Ahlrichs, Phys. Chem. Chem. Phys. 7 (2005) 3297.

19. S. Grimme, S. Ehrlich, L. Goerigk, J. Comput. Chem. 32 (2011) 1456.

20. S. M. Lang, P. Claes, S. Neukermans, E. Janssens, J. Am. Soc. Mass Spectrom. 22 (2011) 1508.

21. E. M. Fernández, A. Vega, L. C. Balbás, J. Chem. Phys. 139 (2013) 214305.

22. V. T. Ngan, E. Janssens, P. Claes, A. Fielicke, M. T. Nguyen, P. Lievens, Phys. Chem. Chem. Phys. 17 (2015) 17584.

23. A. Fielicke, G. Von Helden, G. Meijer, Eur. Phys. J. D 34 (2005) 83.

24. J. Oomens, A. G. G. M. Tielens, B. G. Sartakov, G. Von Helden, G. Meijer, Astrophys. J. 591 (2003) 968.

25. K. R. Asmis, Y. Yang, G. Santambrogio, M. Brümmer, J. R. Roscioli, L. R. McCunn, M. A. Johnson, O. Kühn, Angew. Chem. Int. Ed. 46 (2007) 8691.

26. F. Calvo, Y. Li, D. M. Kiawi, J. M. Bakker, P. Parneix, E. Janssens, Phys. Chem. Chem. Phys. 17 (2015) 1.

27. F. Calvo, P. Parneix, Chem. Phys. Phys. Chem. 13 (2012) 212.

28. D. Bumüller, A.-S. Hehn, E. Waldt, R. Ahlrichs, M. M. Kappes, D. Schooss, J. Phys. Chem. C 121 (2017) 10645.

29. A. A. Mikhailin, O. P. Charkin, N. M. Klimenko, Russ. J. Inorg. Chem. 57 (2012) 528.

30. J. M. Soler, E. Artacho, J. D. Gale, A. García, J. Junquera, P. Ordejón, D. Sánchez-Portal, J. Phys. Condens. Matter 14 (2002) 2745.

31. J. Junquera, Ó. Paz, D. Sánchez-Portal, E. Artacho, Phys. Rev. B - Condens. Matter Mater. Phys. 64 (2001) 23511.

32. M. B. Knickelbein, Annu. Rev. Phys. Chem. 50 (1999) 79.

33. J. Conceicao, R. T. Laaksonen, L.-S. Wang, T. Guo, P. Nordlander, R. E. Smalley, Phys. Rev. B 51 (1995) 4668.

Supplementary Material: The online version of this article offers supplementary material (https://doi.org/10.1515/zpch-2019-1395). 